Research Article

\title{
Nonlinear Vibration Mechanism of the Marine Rotating Machinery with Airbag Isolation Device under Heaving Motion
}

\author{
Xiaolei Du and Ming Li iD \\ Department of Mechanics, Xi'an University of Science and Technology, 710054 Shaanxi, Xi'an, China \\ Correspondence should be addressed to Ming Li; limxust@xust.edu.cn
}

Received 25 June 2020; Revised 28 November 2020; Accepted 22 December 2020; Published 15 January 2021

Academic Editor: Hung Nguyen-Xuan

Copyright (C) 2021 Xiaolei Du and Ming Li. This is an open access article distributed under the Creative Commons Attribution License, which permits unrestricted use, distribution, and reproduction in any medium, provided the original work is properly cited.

\begin{abstract}
In this paper, we put an investigation into the nonlinear vibration mechanism of the marine rotating machinery with an airbag isolation device under heaving motion. First, we consider the effects of mass unbalance and heaving motion and propose a mathematical model of the marine rotating mechanical system with airbag vibration isolation. Then, the multiple-scale method is conducted to analyze the nonlinear dynamic characteristics of the mechanical system; the frequency-response curves are mainly studied under different parameters, such as the heave excitation, rotor speed, and damping; and the numerical method is also introduced to analyze its dynamic behaviors, such as the steady-state response and its corresponding phase diagram, Poincaré section. The dynamic stability of the system is investigated based on the bifurcations and the largest Lyapunov exponent about rotor speed and heaving frequency. The obtained results indicate complex nonlinear characteristics of the system compared to the system without heaving excitation, which can help us fully understand the dynamic characteristics and parametric optimization as well as structural design of the marine mechanical isolation system.
\end{abstract}

\section{Introduction}

As the main power equipment, rotating machinery has been widely used in many important industries such as maritime transportation. However, excessive vibration and annoying noise are inevitable in the rotation machinery due to the complexity of the operation environment. For example, it is seriously affected by the transport motions of a ship in navigation such as the heaving motion. Therefore, various vibration isolation systems were introduced to eliminate their effects, in which an airbag device was usually used according to the characteristic of adjustable stiffness and low natural frequency [1].

In the past decades, some research has been studied on the nonlinear dynamic characteristics of the ship under different motions. For instance, Nayfeh et al. developed a mathematical model of hull movement based on the perturbation-energy approach, which considered the roll and pitch modes [2]; also, researchers have always been committed to how to improve the antioverturning ability of ships in the course of navigation, that is, to study the stability of ships in the wind and waves [3-8]. Bo-Woo Nam et al. analyzed the displacement responses of floating bodies in waves coupled with sloshing-induced internal forces and their effects on sloshing-induced impact loads [3]. Recently, Sanchez et al. studied the dynamic behavior of the FPSOturret system during regular and irregular wave conditions tests and developed an analytical method to predict the maximum yaw angle [7]. Li et al. explored the effects of the yaw error and the wind-wave misalignment on the dynamic characteristics of the floating offshore wind turbine (FOWT) [8]. Moreover, several studies have been considered on the effect of transport motions in airborne rotating machinery [9-12]. For example, Chang-sheng carried out the analysis of flexible rotor systems with unbalanced multidisk, multimass, and multibearing and discussed the influence of space maneuvering flight on the dynamic characteristics of the engine rotor system [9]. In recent years, Lei and Yushu (2016) discussed the dynamic characteristics of the nonlinear aircraft rotor system in Herbst maneuvering flight and 
analyzed the safety of load control [10]. Later, they (2016) studied the nonlinear response of cracked rotor ball bearing system caused by aircraft flight control and analyzed the nonlinear dynamic behavior of the system by using the Runge-Kutta method [11]. For the study on the transport motions in ground-based rotating machinery, Zheng-hao numerically analyzed the random response of a cantilever double-disc rotor system under bearing rotary random excitation, rotor imbalance random excitation, and ground random seismic excitation [13]. Dakel et al. considered the dynamic behavior of an on-board rotor mounted on hydrodynamic journal bearings in the presence of rigid support movements, which considered turbochargers of vehicles and rotating machines subject to seismic excitation [14]. Saeed and El-Ganaini deal with the vibration analysis of a horizontally supported Jeffcott rotor system with a nonlinear restoring force [15]. Some researchers have investigated the behaviors of nonlinear dynamics of the marine rotorbearing system under ship motions. Guanghui studied the nonlinear dynamic response of the marine rotor-bearing system under the pitch and roll motion of a ship [16]. ZhenXing et al. considered the nonlinearity of the oil-film force and tooth force on drive-side and back-side and established the dynamic model of the journal bearing-gear system under the swing motion of the ship [17]. Han analyzed the dynamic characteristics of the ship under heaving motion by using the numerical method [18]. Recently, the research on the dynamic characteristics of the marine rotating machinery system is studied in depth. For instance, Wen investigated the nonlinear dynamic behaviors of the mechanical isolation system coupled with airbag and floating-raft with and without basement excitation in lateral directions, respectively $[19,20]$. Soni analyzed the performance of an AMB used for supporting a marine rotor system under the effect of ship maneuvering by using the finite element method [21].

Heaving motion has a key impact on the stability and safety of a ship [22]. However, the above-mentioned research mainly focused on the nonlinear dynamic responses of the marine rotor system, while the heaving motion of boats is ignored. Thus, their research cannot illustrate the real motion of ships. Besides, less attention is paid on the nonlinear vibration mechanism of the marine rotating machinery with airbag device under heaving motion. Due to the influence of the inertia forces caused by the transport motion, the vibration characteristic of a marine rotorbearing system is changed, and sometimes its movement state may be even turned. Accordingly, the mathematical model and analytical method for the rotor-bearing system under transport motion should be different from the traditional one. Thus, the dynamic effects are necessarily considered. In this paper, we present the model of the marine rotating mechanical system with airbag vibration isolation under the heave excitation. The nonlinear governing equations are derived and solved by the multiscale method. The obtained frequency-response curves of the vibration isolation system are mainly investigated, which provides theoretical advices for the dynamic design and parameter optimization of the marine rotating machinery.
The paper is organized as follows: In Section 2, the model of the rotating machinery with airbag vibration isolation system is formulated. Then, the nonresonance, primary resonance, superharmonic resonance, and subharmonic resonance are mainly studied in Section 3. Next, Section 4 investigates the nonlinear dynamic characteristics of the mechanical system. Finally, the main conclusions are presented in Section 5.

\section{Motion Equation}

Figure 1 depicts a schematic of a rotating mechanical system device. The mechanical system consists of rotating machinery, the airbag isolation device, a damper, and some limits, in which the rotor machine and the airbag are considered as a mass block $m$ and spring with stiffness $k$, respectively. The limiter is also regarded as a spring with a small gap $\delta$ below the mass block. Generally, in mechanic system, $m_{e}$ represents the eccentric mass of the rotor due to misalignment; $e$ is the eccentricity; $d$ is the damping coefficient; $x_{0}$ is the displacement caused by heave, $A_{0}$ is the amplitude of heave, and $\theta_{0}$ is the phase difference between heave and rotor; $x$ means the compression length of the spring; $\omega$ denotes the rotor velocity and $\omega_{z}$ represents the angular frequency of heave; and $k_{1}, k_{2}$, and $k_{3}$ represent the stiffness of the airbag vibration isolation system.

For simplification, the following assumptions upon the system of concern will be used hereinafter: (1) the nonlinear stiffness of the airbag is denoted by the elastic force with cubic nonlinearity [23], expressed as $F_{s}=k_{1} x+\varepsilon k_{2} x^{3}$, and (2) the heave motion is considered as a sinusoidal function here, which is expressed as $x_{0}=A_{0} \sin \left(\omega_{z} t+\theta_{0}\right)$. According to Newton's law, the differential equation of motion can be expressed as

$$
\begin{aligned}
m\left(\ddot{x}+t \ddot{x}_{0}\right)= & -\left(k_{1} x+\varepsilon k_{2} x^{3}\right)-\mathrm{d} \dot{x}+m_{e} e \omega^{2} \sin \omega t \\
& +m g-k_{3} \vartheta(x),
\end{aligned}
$$

with $\vartheta(x)=\left\{\begin{array}{ll}x-\delta, & x \geq \delta \\ 0, & x<\delta\end{array}\right.$, which can be rewritten in $\ddot{x}+\frac{d}{m} \dot{x}+\frac{k_{1}}{m} x+\varepsilon \frac{k_{2}}{m} x^{3}+\frac{k_{3}}{m} \vartheta(x)=\frac{m_{e}}{m} e \omega^{2} \sin \omega t+g-\ddot{x}_{0}$.

To understand the mechanism of the microvibration of the system, we consider the case of the stopper not been triggered, that is, the situation that the compression length is lower than the stopper clearance.

Then, the nondimensional nonlinear governing equation can be obtained based on Table 1 , where $c$ is the bearing clearances. For the convenience of analysis on heaving motion, we assume that $\theta_{0}=0$. Then, (2) can be rewritten as

$$
\begin{aligned}
c \omega^{2} X^{\prime \prime}+c \omega \frac{d}{m} X^{\prime}+\frac{k_{1}}{m} c X+\varepsilon \frac{k_{2}}{m} c^{3} X^{3}= & \frac{m_{e}}{m} e \omega^{2} \sin \tau \\
& +g-c \omega^{2} \bar{X}_{0}^{\prime \prime} .
\end{aligned}
$$




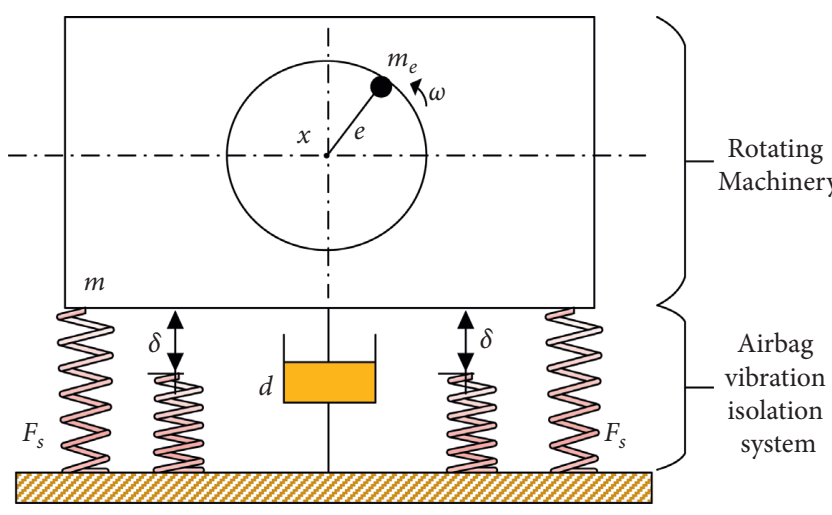

FIGURE 1: Schematic diagram of rotating mechanical system with airbag vibration isolation.

TABLE 1: The expression of nondimensional parameter.

\begin{tabular}{lc}
\hline Parameter description & Expression \\
\hline Nondimensional frequency ratio $\eta$ & $\eta=\omega / \omega_{z}$ \\
Nondimensional time $\tau$ & $\tau=\omega t$ \\
Nondimensional mass ratio $h$ & $h=m_{e} / m$ \\
Nondimensional eccentricity $\alpha$ & $\alpha=e / c$ \\
Nondimensional displacement of rotor $X$ & $X=x / c$ \\
Nondimensional displacement of ship $X_{0}$ & $X_{0}=x_{0} / c$ \\
Nondimensional speed $\Omega_{0}$ & $\Omega_{0}=\sqrt{c \omega^{2} / g}$ \\
Nondimensional spring stiffness $k_{1}$ & $\underline{k}_{1}=c k_{1} / \mathrm{mg}$ \\
Nondimensional coefficient_k$k_{2}$ & $k_{2-}=c^{3} k_{2} / \mathrm{mg}$ \\
Nondimensional damping $d$ & $d=d / m \omega$ \\
Nondimensional heaving amplitude $\bar{A}$ & $A=A_{0} / c$ \\
\hline
\end{tabular}

We define $\dot{x}=\mathrm{d} x / \mathrm{d} t \quad \dot{x}_{0}=\mathrm{d} x_{0} / \mathrm{d} t \quad X^{\prime}=\mathrm{d} X / \mathrm{d} \tau \quad X_{0}^{\prime}=$ $\mathrm{d} X_{0} / \mathrm{d} \tau$, the motion equation's dimensionless expression is obtained as follows:

$$
X^{\prime \prime}+\overline{\mathrm{d}} X^{\prime}+\frac{\bar{k}_{1}}{\Omega_{0}^{2}} X+\varepsilon \frac{\bar{k}_{2}}{\Omega_{0}^{2}} X^{3}=h \alpha \sin \tau+\frac{1}{\Omega_{0}^{2}}-\bar{X}_{0}^{\prime \prime} .
$$

We let $\alpha=\varepsilon \alpha_{0}$ and $\bar{d}=\varepsilon \mu$ since the values of $\alpha$ and $\bar{d}$ are much smaller compared to $\bar{A} / \eta^{2}$ in practice. Then, to simplify the equation, we also define $\omega_{1}^{2}=\bar{k}_{1} / \Omega_{0}^{2}, b=\bar{k}_{2} / \Omega_{0}^{2}$, $F=\bar{A} / \eta^{2}$. Then, (4) can be cast into the following nondimensional form:

$$
X^{\prime \prime}+\omega_{1}^{2} X=-\varepsilon \mu X^{\prime}-\varepsilon b X^{3}+\varepsilon h \alpha_{0} \sin \tau+\frac{1}{\Omega_{0}^{2}}+F \sin \frac{\tau}{\eta} .
$$

\section{Theoretical Analysis}

Here, to discuss the vibration mechanism and characteristics of the system, the method of multiple scales (MMS) is introduced for the approximation solution of the nonlinear vibration.

Let $X(\tau, \varepsilon)=X_{0}\left(T_{0}, T_{1}\right)+\varepsilon X_{1}\left(T_{0}, T_{1}\right)$, where $\varepsilon$ is a small perturbation characterizing the amplitude of the motion and $T_{0}=\tau, T_{1}=\varepsilon \tau$. Define partial differential operator $D_{n}=\partial / \partial T_{n}$ and substitute it into (5); we can get

$$
\left\{\begin{array}{l}
D_{0}^{2} X_{0}+\omega_{1}^{2} X_{0}=\frac{1}{\Omega_{0}^{2}}+F \sin \frac{T_{0}}{\eta}, \\
D_{0}^{2} X_{1}+\omega_{1}^{2} X_{1}=-\mu D_{0} X_{0}-2 D_{0} D_{1} X_{0}-b X_{0}^{3}+\alpha_{0} h \sin T_{0} .
\end{array}\right.
$$

Then, the solution of $X_{0}$ is obtained:

$$
X_{0}=A \exp \left(i \omega_{1} T_{0}\right)+\Lambda \exp \left(i \frac{T_{0}}{\eta}\right)+\frac{1}{2 \omega_{1}^{2} \Omega_{0}^{2}}+c c,
$$

where $\Lambda=-F \eta^{2} i / 2\left(\eta^{2} \omega_{1}^{2}-1\right)$.

Substituting (7) into the second equation of equation (6), it yields

$$
\begin{array}{r}
D_{0}^{2} X_{1}+\omega_{1}^{2} X_{1}=-\left(3 A^{2} \bar{A} b+2 \omega_{1} i D_{1} A+\mu \omega_{1} i A+6 A \Lambda \bar{\Lambda} b+\frac{3 b A}{\omega_{1}^{4} \Omega_{0}^{4}}\right) \exp \left(i \omega_{1} T_{0}\right) \\
-\frac{\alpha_{0} h}{2} i \exp \left(i T_{0}\right)-\frac{3 b A^{2}}{\omega_{1}^{2} \Omega_{0}^{2}} \exp \left(2 i \omega_{1} T_{0}\right)-A^{3} b \exp \left(3 i \omega_{1} T_{0}\right)-\frac{6 b A \Lambda}{\omega_{1}^{2} \Omega_{0}^{2}} \exp \left(i \omega_{1} T_{0}+i \frac{T_{0}}{\eta}\right) \\
-3 A \Lambda^{2} b \exp \left(i \omega_{1} T_{0}+2 i \frac{T_{0}}{\eta}\right)-\frac{6 b \bar{A} \Lambda}{\omega_{1}^{2} \Omega_{0}^{2}} \exp \left(i \frac{T_{0}}{\eta}-i \omega_{1} T_{0}\right)-3 A \bar{\Lambda}^{2} b \exp \left(i \omega_{1} T_{0}-2 i \frac{T_{0}}{\eta}\right) \\
-3 A^{2} \Lambda b \exp \left(2 i \omega_{1} T_{0}+i \frac{T_{0}}{\eta}\right)-3 \bar{A}^{2} \Lambda b \exp \left(i \frac{T_{0}}{\eta}-2 i \omega_{1} T_{0}\right)-\left(6 A \bar{A} \Lambda b+3 \Lambda^{2} \bar{\Lambda} b+\frac{3 b \Lambda}{\omega_{1}^{4} \Omega_{0}^{4}}+\frac{\mu}{\eta} i \Lambda\right) \exp \left(i \frac{T_{0}}{\eta}\right) \\
-\frac{3 b \Lambda^{2}}{\omega_{1}^{2} \Omega_{0}^{2}} \exp \left(2 i \frac{T_{0}}{\eta}\right)-\Lambda^{3} b \exp \left(3 i \frac{T_{0}}{\eta}\right)-\frac{3 b A \bar{A}}{\omega_{1}^{2} \Omega_{0}^{2}}-\frac{3 b \Lambda \bar{\Lambda}}{\omega_{1}^{2} \Omega_{0}^{2}}-\frac{b}{2 \omega_{1}^{6} \Omega_{0}^{6}}+c c,
\end{array}
$$


where $\mathrm{cc}$ is the complex conjugate of the preceding terms.

3.1. Nonresonance Case. Eliminate the secular term in (8); that is,

$$
3 A^{2} \bar{A} b+2 \omega_{1} i D_{1} A+\mu \omega_{1} i A+6 A \Lambda \bar{\Lambda} b+\frac{3 b A}{\omega_{1}^{4} \Omega_{0}^{4}}=0 .
$$

Let $A=1 / 2 a\left(T_{1}\right) \exp \left[i \beta\left(T_{1}\right)\right]$, substitute $A$ into (9), and separate the real and imaginary parts of the equation:

$$
\left\{\begin{array}{l}
\frac{3}{8} b a^{3}-\omega_{1} a \dot{\beta}+3 a b \Lambda \bar{\Lambda}+\frac{3 b a}{2 \omega_{1}^{4} \Omega_{0}^{4}}=0, \\
\omega_{1} \dot{a}=-\frac{1}{2} \mu a \omega_{1} .
\end{array}\right.
$$

Then, $a$ and $\beta$ are obtained:

$$
\left\{\begin{array}{l}
a=a_{0} \exp \left(-\frac{1}{2} \mu T_{1}\right), \\
\beta=-\frac{3 b a_{0}^{2}}{8 \mu \omega_{1}} \exp \left(-\mu T_{1}\right)+\left(\frac{3 b \Lambda \bar{\Lambda}}{\omega_{1}}+\frac{3 b}{2 \omega_{1}^{5} \Omega_{0}^{4}}\right) T_{1}+\beta_{0} .
\end{array}\right.
$$

Hence, the first approximate solution of (5) is

$$
X=a_{0} \exp \left(-\frac{1}{2} \mu \varepsilon \tau\right) \cos \left[\omega_{1} \tau-\frac{3 b a_{0}^{2}}{8 \mu \omega_{1}} \exp (-\mu \varepsilon \tau)+\left(\frac{3 b \Lambda \bar{\Lambda}}{\omega_{1}}+\frac{3 b}{2 \omega_{1}^{5} \Omega_{0}^{4}}\right) \varepsilon \tau+\beta_{0}\right]+\frac{F \eta^{2}}{\eta^{2} \omega_{1}^{2}-1} \sin \left(\frac{\tau}{\eta}\right)+\frac{1}{\omega_{1}^{2} \Omega_{0}^{2}}
$$

$a_{0}$ and $\beta_{0}$ are two constants determined by the initial conditions. In the case of nonresonance, the amplitude of the free vibration decays exponentially with time. The steadystate response is affected by heave motion.

3.2. Primary Resonance Case. The main analysis is conducted to the primary resonance of the system caused by the unbalanced force. Then, we define a frequency tuning factor $\sigma$, when $1=\omega_{1}+\varepsilon \sigma, \exp \left(i T_{0}\right)=\exp \left(i \omega_{1} T_{0}+i \sigma T_{1}\right)$. Hence, the secular term can be expressed as follows:

$3 A^{2} \bar{A} b+2 \omega_{1} i D_{1} A+\mu \omega_{1} i A+6 A \Lambda \bar{\Lambda} b+\frac{3 b A}{\omega_{1}^{4} \Omega_{0}^{4}}+\frac{\alpha_{0} h}{2} i \exp \left(i \sigma T_{1}\right)=0$.

Let $A=1 / 2 a\left(T_{1}\right) \exp \left[i \beta\left(T_{1}\right)\right]$, substitute it into (13), and separate the real and imaginary parts of the equation:

$$
\left\{\begin{array}{l}
\dot{a}=-\frac{1}{2} \mu a-\frac{\alpha_{0} h}{2 \omega_{1}} \cos \left(\sigma T_{1}-\beta\right), \\
\dot{a} \dot{\beta}=\frac{3 b a^{3}}{8 \omega_{1}}+\frac{3 a b \Lambda \bar{\Lambda}}{\omega_{1}}+\frac{3 b a}{2 \omega_{1}^{5} \Omega_{0}^{4}}-\frac{\alpha_{0} h}{2 \omega_{1}} \sin \left(\sigma T_{1}-\beta\right),
\end{array}\right.
$$

where $a$ and $\beta$ are determined by (14). Then, (14) can be transformed into an autonomous system by setting $\gamma=\sigma T_{1}-\beta$, and thereby obtaining

$$
\left\{\begin{array}{l}
\dot{a}=-\frac{1}{2} \mu a-\frac{\alpha_{0} h}{2 \omega_{1}} \cos \gamma, \\
a \dot{\gamma}=a \sigma-\frac{3 b a^{3}}{8 \omega_{1}}-\frac{3 a b \Lambda \bar{\Lambda}}{\omega_{1}}-\frac{3 b a}{2 \omega_{1}^{5} \Omega_{0}^{4}}+\frac{\alpha_{0} h}{2 \omega_{1}} \sin \gamma .
\end{array}\right.
$$

Therefore, for the first approximation,

$$
X=a \cos (\tau-\gamma)+\frac{F \eta^{2}}{\left.\eta^{2}(1-\varepsilon \sigma)^{2}-1\right)} \sin \frac{\tau}{\eta}+\frac{1}{(1-\varepsilon \sigma)^{2} \Omega_{0}^{2}} .
$$

To characterize the stability of the solution, the Jacobian matrix of the system is solved, and the autonomous differential equation is linearized at the steady solution to obtain the corresponding characteristic equation. Thus, the condition leads to

$$
\left|\begin{array}{cc}
-\frac{1}{2} \mu-\lambda & -a_{0}\left(\sigma-\frac{3 b a_{0}^{2}}{8 \omega_{1}}-\frac{3 b \Lambda \bar{\Lambda}}{\omega_{1}}-\frac{3 b}{2 \omega_{1}^{5} \Omega_{0}^{4}}\right) \\
\frac{1}{a_{0}}\left(\sigma-\frac{3 b a_{0}^{2}}{8 \omega_{1}}-\frac{3 b \Lambda \bar{\Lambda}}{\omega_{1}}-\frac{3 b}{2 \omega_{1}^{5} \Omega_{0}^{4}}\right) & -\frac{1}{2} \mu-\lambda
\end{array}\right|=0
$$


To solve (17),

$$
\lambda^{2}+\mu \lambda+\frac{1}{4} \mu^{2}+\left(\sigma-\frac{3 b a_{0}^{2}}{8 \omega_{1}}-\frac{3 b \Lambda \bar{\Lambda}}{\omega_{1}}-\frac{3 b}{2 \omega_{1}^{5} \Omega_{0}^{4}}\right)^{2}=0
$$

For the case of $\mu>0,1 / 4 \mu^{2}+\left(\sigma-\left(3 b a_{0}^{2} / 8 \omega_{1}\right)\right.$ $\left.-\left(3 b \Lambda \bar{\Lambda} / \omega_{1}\right)-\left(3 b / 2 \omega_{1}^{5} \Omega_{0}^{4}\right)\right)^{2}>0$, which indicates the stability condition of the steady solution is satisfied. The steadystate response corresponds to $\dot{a}=\dot{\gamma}=0$, corresponding to the solution of

$$
\left\{\begin{array}{l}
\frac{1}{2} \mu a=-\frac{\alpha_{0} h}{2 \omega_{1}} \cos \gamma \\
a \sigma-\frac{3 b a^{3}}{8 \omega_{1}}-\frac{3 a b \Lambda \bar{\Lambda}}{\omega_{1}}-\frac{3 b a}{2 \omega_{1}^{5} \Omega_{0}^{4}}=-\frac{\alpha_{0} h}{2 \omega_{1}} \sin \gamma .
\end{array}\right.
$$

$$
3 A^{2} \bar{A} b+2 \omega_{1} i D_{1} A+\mu \omega_{1} i A+6 A \Lambda \bar{\Lambda} b+\frac{3 b A}{\omega_{1}^{4} \Omega_{0}^{4}}+\frac{3 b \Lambda^{2}}{\omega_{1}^{2} \Omega_{0}^{2}} \exp \left(i \sigma T_{1}\right)=0
$$

Let $A=1 / 2 a\left(T_{1}\right) \exp \left[i \beta\left(T_{1}\right)\right]$, substitute the above formula, and separate the real and virtual parts of the equation:

$$
\left\{\begin{array}{l}
\dot{a}=-\frac{1}{2} \mu a-\frac{3 b \Lambda^{2}}{\omega_{1}^{3} \Omega_{0}^{2}} \sin \left(\sigma T_{1}-\beta\right), \\
a \dot{\beta}=\frac{3 b a^{3}}{8 \omega_{1}}+\frac{3 a b \Lambda \bar{\Lambda}}{\omega_{1}}+\frac{3 b a}{2 \omega_{1}^{5} \Omega_{0}^{4}}+\frac{3 b \Lambda^{2}}{\omega_{1}^{3} \Omega_{0}^{2}} \cos \left(\sigma T_{1}-\beta\right),
\end{array}\right.
$$

where $\alpha$ and $\beta$ are determined by (22). Then, (22) can be transformed into an autonomous system by setting $\gamma=\sigma T_{1}-\beta$, and thereby obtaining
The response equations of amplitude frequency and phase frequency of the main resonance of the system are obtained by eliminating $\gamma$ in (19):

$$
\begin{gathered}
\left(a \sigma-\frac{3 b a^{3}}{8 \omega_{1}}-\frac{3 F^{2} \eta^{4} b a}{4 \omega_{1}\left(\eta^{2} \omega_{1}^{2}-1\right)^{2}}-\frac{3 b a}{2 \omega_{1}^{5} \Omega_{0}^{4}}\right)^{2}+\left(\frac{\mu a}{2}\right)^{2}=\left(\frac{\alpha_{0} h}{2 \omega_{1}}\right)^{2} \\
\tan \gamma=\frac{2 \sigma}{\mu}-\frac{3 b a^{2}}{4 \mu \omega_{1}}-\frac{3 F^{2} \eta^{4} b}{2 \mu \omega_{1}\left(\eta^{2} \omega_{1}^{2}-1\right)^{2}}-\frac{3 b}{\mu \omega_{1}^{5} \Omega_{0}^{4}} .
\end{gathered}
$$

3.3. Superharmonic Resonance, $2 / \eta \approx \omega_{1}$. The superharmonic resonance happens when $2 / \eta=\omega_{1}+\varepsilon \sigma, \exp \left(2 i T_{0} / \eta\right)=\exp$ $\left(i \omega_{1} T_{0}+i \sigma T_{1}\right)$. Then, the secular term can be expressed as follows:

$$
\left\{\begin{array}{l}
\dot{a}=-\frac{1}{2} \mu a-\frac{3 b \Lambda^{2}}{\omega_{1}^{3} \Omega_{0}^{2}} \sin \gamma \\
a \dot{\gamma}=a \sigma-\frac{3 b a^{3}}{8 \omega_{1}}-\frac{3 a b \Lambda \bar{\Lambda}}{\omega_{1}}-\frac{3 b a}{2 \omega_{1}^{5} \Omega_{0}^{4}}-\frac{3 b \Lambda^{2}}{\omega_{1}^{3} \Omega_{0}^{2}} \cos \gamma
\end{array}\right.
$$

Here, the approximate solution is

$$
X=\operatorname{acos}\left(\frac{2}{\eta} \tau-\gamma\right)+\frac{4 F \eta^{2}}{3-2 \varepsilon \sigma \eta^{2}-\varepsilon^{2} \sigma^{2} \eta^{2}} \sin \frac{\tau}{\eta}+\frac{1}{((2 / \eta)-\varepsilon \sigma)^{2} \Omega_{0}^{2}} .
$$

In order to characterize the stability of the solution, the condition is as follows:

$$
\left|\begin{array}{cc}
-\frac{1}{2} \mu-\lambda & -a_{0}\left(\sigma-\frac{3 b a_{0}^{2}}{8 \omega_{1}}-\frac{3 b \Lambda \bar{\Lambda}}{\omega_{1}}-\frac{3 b}{2 \omega_{1}^{5} \Omega_{0}^{4}}\right) \\
\frac{1}{a_{0}}\left(\sigma-\frac{3 b a_{0}^{2}}{8 \omega_{1}}-\frac{3 b \Lambda \bar{\Lambda}}{\omega_{1}}-\frac{3 b}{2 \omega_{1}^{5} \Omega_{0}^{4}}\right) & -\frac{1}{2} \mu-\lambda
\end{array}\right|=0
$$

which could also be expressed as

$$
\lambda^{2}+\mu \lambda+\frac{1}{4} \mu^{2}+\left(\sigma-\frac{3 b a_{0}^{2}}{8 \omega_{1}}-\frac{3 b \Lambda \bar{\Lambda}}{\omega_{1}}-\frac{3 b}{2 \omega_{1}^{5} \Omega_{0}^{4}}\right)^{2}=0 .
$$

For the case of $\mu>0, \quad 1 / 4 \mu^{2}+\left(\sigma-\left(3 b a_{0}^{2} /\right.\right.$ $\left.\left.8 \omega_{1}\right)-\left(3 b \Lambda \bar{\Lambda} / \omega_{1}\right)-\left(3 b / 2 \omega_{1}^{5} \Omega_{0}^{4}\right)\right)^{2}>0$, which shows that the stability condition of the steady solution is satisfied. The steady-state response corresponds to $\dot{a}=\dot{\gamma}=0$; then, eliminating $\gamma$ leads to the frequency-response equation:

$$
\left(a \sigma-\frac{3 b a^{3}}{8 \omega_{1}}-\frac{3 F^{2} \eta^{4} b a}{4 \omega_{1}\left(\eta^{2} \omega_{1}^{2}-1\right)^{2}}-\frac{3 b a}{2 \omega_{1}^{5} \Omega_{0}^{4}}\right)^{2}+\left(\frac{\mu a}{2}\right)^{2}=\left(\frac{3 F^{2} \eta^{4} b}{4\left(\eta^{2} \omega_{1}^{2}-1\right)^{2} \omega_{1}^{3} \Omega_{0}^{2}}\right)^{2} .
$$


3.4. Superharmonic Resonance, $3 / \eta \approx \omega_{1}$. The superharmonic resonance could also happen when $3 / \eta=\omega_{1}+\varepsilon \sigma$, $\exp \left(3 i T_{0} / \eta\right)=\exp \left(i \omega_{1} T_{0}+i \sigma T_{1}\right)$. Then, the secular term can be expressed as follows:

$3 A^{2} \bar{A} b+2 \omega_{1} i D_{1} A+\mu \omega_{1} i A+6 A \Lambda \bar{\Lambda} b+\frac{3 b A}{\omega_{1}^{4} \Omega_{0}^{4}}+\Lambda^{3} b \exp \left(i \sigma T_{1}\right)=0$.

Then, the approximate solution is

$$
X=a \cos \left(\frac{3}{\eta} \tau-\gamma\right)+\frac{9 F}{8 \omega_{1}^{2}-2 \varepsilon \sigma \omega_{1}-\varepsilon^{2} \sigma^{2}} \sin \frac{\tau}{\eta}+\frac{1}{((3 / \eta)-\varepsilon \sigma)^{2} \Omega_{0}^{2}} .
$$

Compared to the linear case, although there is positive damping, the free vibration does not decay to zero and the nonlinearity adjusts the frequency of the free vibration term which makes it exactly three times the excitation frequency. Thus, the response is periodic:

$$
\left|\begin{array}{cc}
-\frac{1}{2} \mu-\lambda & -a_{0}\left(\sigma-\frac{3 b a_{0}^{2}}{8 \omega_{1}}-\frac{3 b \Lambda \bar{\Lambda}}{\omega_{1}}-\frac{3 b}{2 \omega_{1}^{5} \Omega_{0}^{4}}\right) \\
\frac{1}{a_{0}}\left(\sigma-\frac{3 b a_{0}^{2}}{8 \omega_{1}}-\frac{3 b \Lambda \bar{\Lambda}}{\omega_{1}}-\frac{3 b}{2 \omega_{1}^{5} \Omega_{0}^{4}}\right) & -\frac{1}{2} \mu-\lambda
\end{array}\right|=0
$$

which could also be expressed as

$$
\lambda^{2}+\mu \lambda+\frac{1}{4} \mu^{2}+\left(\sigma-\frac{3 b a_{0}^{2}}{8 \omega_{1}}-\frac{3 b \Lambda \bar{\Lambda}}{\omega_{1}}-\frac{3 b}{2 \omega_{1}^{5} \Omega_{0}^{4}}\right)^{2}=0 .
$$

For the case of $\mu>0, \quad 1 / 4 \mu^{2}+\left(\sigma-\left(3 b a_{0}^{2} / 8 \omega_{1}\right)\right.$ $\left.-\left(3 b \Lambda \bar{\Lambda} / \omega_{1}\right)-\left(3 b / 2 \omega_{1}^{5} \Omega_{0}^{4}\right)\right)^{2}>0$, which means that the stability condition of the steady solution is satisfied. The steadystate response corresponds to $\dot{a}=\dot{\gamma}=0$, which corresponds to the solution of

$$
\left\{\begin{array}{l}
\frac{1}{2} \mu a=-\frac{\Lambda^{3} b}{\omega_{1}} \cos \gamma, \\
a \sigma-\frac{3 b a^{3}}{8 \omega_{1}}-\frac{3 a b \Lambda \bar{\Lambda}}{\omega_{1}}-\frac{3 b a}{2 \omega_{1}^{5} \Omega_{0}^{4}}=-\frac{\Lambda^{3} b}{\omega_{1}} \sin \gamma,
\end{array}\right.
$$

and by eliminating $\gamma$, the frequency-response equation of the superharmonic resonance of the system is obtained:

$$
\left(a \sigma-\frac{3 b a^{3}}{8 \omega_{1}}-\frac{3 F^{2} \eta^{4} b a}{4 \omega_{1}\left(\eta^{2} \omega_{1}^{2}-1\right)^{2}}-\frac{3 b a}{2 \omega_{1}^{5} \Omega_{0}^{4}}\right)^{2}+\left(\frac{\mu a}{2}\right)^{2}=\left(\frac{F^{3} \eta^{6} b}{8 \omega_{1}\left(\eta^{2} \omega_{1}^{2}-1\right)^{3}}\right)^{2}
$$

3.5. Subharmonic Resonance, $1 / \eta \approx 2 \omega_{1}$. The subharmonic resonance happens when $1 / \eta=2 \omega_{1}+\varepsilon \sigma$, $\exp \left(i T_{0} / \eta-i \omega_{1} T_{0}\right)=\exp \left(i \omega_{1} T_{0}+i \sigma T_{1}\right)$. Then, the secular term can be expressed as follows:

$$
3 A^{2} \bar{A} b+2 \omega_{1} i D_{1} A+\mu \omega_{1} i A+6 A \Lambda \bar{\Lambda} b+\frac{3 b A}{\omega_{1}^{4} \Omega_{0}^{4}}+\frac{6 b \bar{A} \Lambda}{\omega_{1}^{2} \Omega_{0}^{2}} \exp \left(i \sigma T_{1}\right)=0 .
$$

Thus, the approximate solution can be expressed as

$$
X=\operatorname{acos}\left[\frac{1}{2}\left(\frac{\tau}{\eta}-\gamma\right)\right]+\frac{F \eta^{2}}{\eta^{2} \omega_{1}^{2}-1} \sin \frac{\tau}{\eta}+\frac{1}{\omega_{1}^{2} \Omega_{0}^{2}} .
$$

The characteristic equation of the system is

$$
\lambda^{2}+\lambda \mu+\frac{3 a b}{2 \omega_{1}}\left(\frac{3 a^{3} b}{8 \omega_{1}}+\frac{3 a b F^{2} \eta^{4}}{4 \omega_{1}\left(\eta^{2} \omega_{1}^{2}-1\right)^{2}}+\frac{3 a b}{2 \omega_{1}^{5} \Omega_{0}^{4}}-\frac{a \sigma}{2}\right)=0 .
$$

In this case, for $\mu>0$, the system is asymptotic stable if $3 a b / 2 \omega_{1}\left(\left(3 a^{3} b / 8 \omega_{1}\right)+\left(3 a b F^{2} \eta^{4} / 4 \omega_{1}\left(\eta^{2} \omega_{1}^{2}-1\right)^{2}\right)+(3 a b /\right.$ $\left.\left.2 \omega_{1}^{5} \Omega_{0}^{4}\right)-(a \sigma / 2)\right)>0$,

$$
\sigma<\frac{3 a^{2} b}{4 \omega_{1}}+\frac{3 b F^{2} \eta^{4}}{2 \omega_{1}\left(\eta^{2} \omega_{1}^{2}-1\right)^{2}}+\frac{3 b}{\omega_{1}^{5} \Omega_{0}^{4}} .
$$

Then, the frequency-response equation of $1 / 2$ order subharmonic resonance of the system is obtained: 


$$
\left(\frac{1}{2} \sigma-\frac{3 a^{2} b}{8 \omega_{1}}-\frac{3 F^{2} \eta^{4} b}{4 \omega_{1}\left(\eta^{2} \omega_{1}^{2}-1\right)^{2}}-\frac{3 b}{2 \omega_{1}^{5} \Omega_{0}^{4}}\right)^{2}+\left(\frac{\mu}{2}\right)^{2}=\left(\frac{3 b F \eta^{2}}{2 \omega_{1}^{3} \Omega_{0}^{2}\left(\eta^{2} \omega_{1}^{2}-1\right)}\right)^{2} .
$$

This is a quadratic algebraic equation for the steady solution of amplitude $a$, which can be written as $a^{4}-2 p a^{2}+q=0$, where

$$
\begin{aligned}
& p=\frac{12 \omega_{1}}{9 b}\left(\sigma-\frac{3 F^{2} \eta^{4} b}{2 \omega_{1}\left(\eta^{2} \omega_{1}^{2}-1\right)^{2}}-\frac{3 b}{\omega_{1}^{5} \Omega_{0}^{4}}\right), \\
& q=\frac{16 \omega_{1}^{2} \mu^{2}}{9 b^{2}}-\left(\frac{4 F \eta^{2}}{\omega_{1}^{2} \Omega_{0}^{2}\left(\eta^{2} \omega_{1}^{2}-1\right)}\right)^{2}+\frac{64 \omega_{1}^{2}}{9 b^{2}}\left(\frac{1}{2} \sigma-\frac{3 F^{2} \eta^{4} b}{4 \omega_{1}\left(\eta^{2} \omega_{1}^{2}-1\right)^{2}}-\frac{3 b}{2 \omega_{1}^{5} \Omega_{0}^{4}}\right)^{2} .
\end{aligned}
$$

It yields $a^{2}=p \pm \sqrt{p^{2}-q}$; thus, the necessary conditions of $1 / 2$ subharmonic resonance are $p>0, q>0, p^{2} \geq q$, which are

$$
\begin{aligned}
\frac{12 \omega_{1}}{9 b}\left(\sigma-\frac{3 F^{2} \eta^{4} b}{2 \omega_{1}\left(\eta^{2} \omega_{1}^{2}-1\right)^{2}}-\frac{3 b}{\omega_{1}^{5} \Omega_{0}^{4}}\right)> & 0 \frac{16 \omega_{1}^{2} \mu^{2}}{9 b^{2}}+\left(\frac{4 \omega_{1} \sigma}{3 b}-\frac{2 F^{2} \eta^{4}}{\left(\eta^{2} \omega_{1}^{2}-1\right)^{2}}-\frac{4}{2 \omega_{1}^{4} \Omega_{0}^{4}}\right)^{2}>\frac{16 F^{2} \eta^{4}}{\omega_{1}^{4} \Omega_{0}^{4}\left(\eta^{2} \omega_{1}^{2}-1\right)^{2}}, \\
& \cdot\left[\frac{12 \omega_{1}}{9 b}\left(\sigma-\frac{3 F^{2} \eta^{4} b}{2 \omega_{1}\left(\eta^{2} \omega_{1}^{2}-1\right)^{2}}-\frac{3 b}{\omega_{1}^{5} \Omega_{0}^{4}}\right)\right]^{2} \geq \frac{16 \omega_{1}^{2} \mu^{2}}{9 b^{2}}-\left(\frac{4 F \eta^{2}}{\omega_{1}^{2} \Omega_{0}^{2}\left(\eta^{2} \omega_{1}^{2}-1\right)}\right)^{2} \\
& +\frac{64 \omega_{1}^{2}}{9 b^{2}}\left(\frac{1}{2} \sigma-\frac{3 F^{2} \eta^{4} b}{4 \omega_{1}\left(\eta^{2} \omega_{1}^{2}-1\right)^{2}}-\frac{3 b}{2 \omega_{1}^{5} \Omega_{0}^{4}}\right)^{2} .
\end{aligned}
$$

It can be observed from (33) and (34) that the subharmonic resonance of $1 / 2$ order occurs in the frequency band slightly higher than the excitation frequency, and the damping can also effectively suppress the subharmonic resonance of $1 / 2$ order; if the above inequalities are not satisfied, there is only the steady solution.
3.6. Subharmonic Resonance, $1 / \eta \approx 3 \omega_{1}$. The subharmonic resonance also happens when $1 / \eta=3 \omega_{1}+\varepsilon \sigma$, $\exp \left(i T_{0} / \eta-2 i \omega_{1} T_{0}\right)=\exp \left(i \omega_{1} T_{0}+i \sigma T_{1}\right)$. Then, the secular term can be expressed:

$$
3 A^{2} \bar{A} b+2 \omega_{1} i D_{1} A+\mu \omega_{1} i A+6 A \Lambda \bar{\Lambda} b+\frac{3 b A}{\omega_{1}^{4} \Omega_{0}^{4}}+3 \bar{A}^{2} \Lambda b \exp \left(i \sigma T_{1}\right)=0
$$

The approximate solution is

$$
X=a \cos \left[\frac{1}{3}\left(\frac{\tau}{\eta}-\gamma\right)\right]+\frac{F \eta^{2}}{\eta^{2} \omega_{1}^{2}-1} \sin \frac{\tau}{\eta}+\frac{1}{\omega_{1}^{2} \Omega_{0}^{2}} .
$$

The condition of the stability of the solution is obtained as follows:

$$
\lambda^{2}+\frac{\mu}{2} \lambda-\frac{\mu^{2}}{2}+\left(\frac{1}{3} a \sigma-\frac{3 a^{3} b}{8 \omega_{1}}-\frac{3 a b F^{2} \eta^{4}}{2 \omega_{1}\left(\eta^{2} \omega_{1}^{2}-1\right)^{2}}-\frac{3 b a}{2 \omega_{1}^{5} \Omega_{0}^{4}}\right)\left(\frac{\sigma}{a}+\frac{9 a b}{8 \omega_{1}}-\frac{9 b F^{2} \eta^{4}}{2 a \omega_{1}\left(\eta^{2} \omega_{1}^{2}-1\right)^{2}}-\frac{9 b}{2 a \omega_{1}^{5} \Omega_{0}^{4}}\right)=0 .
$$


For $\mu>0$, thus the sufficient and necessary conditions of $1 / 3$ order subharmonic resonance are

$$
-\frac{\mu^{2}}{2}+\left(\frac{1}{3} a \sigma-\frac{3 a^{3} b}{8 \omega_{1}}-\frac{3 a b F^{2} \eta^{4}}{2 \omega_{1}\left(\eta^{2} \omega_{1}^{2}-1\right)^{2}}-\frac{3 b a}{2 \omega_{1}^{5} \Omega_{0}^{4}}\right)\left(\frac{\sigma}{a}+\frac{9 a b}{8 \omega_{1}}-\frac{9 b F^{2} \eta^{4}}{2 a \omega_{1}\left(\eta^{2} \omega_{1}^{2}-1\right)^{2}}-\frac{9 b}{2 a \omega_{1}^{5} \Omega_{0}^{4}}\right)>0 .
$$

According to the solution of the steady-state response, the characteristic equations of amplitude frequency of $1 / 3$ order subharmonic resonance of the system are obtained:

$$
\left.\left(\frac{1}{2} \sigma-\frac{3 a^{2} b}{8 \omega_{1}}-\frac{3 F^{2} \eta^{4} b}{4 \omega_{1}\left(\eta^{2} \omega_{1}^{2}-1\right)^{2}}-\frac{3 b}{2 \omega_{1}^{5} \Omega_{0}^{4}}\right)^{2}+\left(\frac{\mu}{2}\right)^{2}=\left(\frac{3 a b F \eta^{2}}{8 \omega_{1}\left(\eta^{2} \omega_{1}^{2}-1\right)}\right)^{2} . " \text { boolean }(. / / m f r a c)^{\prime \prime}, " \text { true" }\right)>
$$

This is a quadratic algebraic equation for the steady solution of amplitude $a$, which can be written as $a^{4}-2 p a^{2}+q=0$, where

$$
\begin{aligned}
& p=\frac{F^{2} \eta^{4}}{2\left(\eta^{2} \omega_{1}^{2}-1\right)^{2}}+\frac{4 \omega_{1} \sigma}{3 b}-\frac{2 F^{2} \eta^{4}}{\left(\eta^{2} \omega_{1}^{2}-1\right)^{2}}-\frac{4}{\omega_{1}^{4} \Omega_{0}^{4}} \\
& q=\left(\frac{8 \omega_{1}}{3 b}\right)^{2}\left(\frac{1}{2} \sigma-\frac{3 F^{2} \eta^{4} b}{4 \omega_{1}\left(\eta^{2} \omega_{1}^{2}-1\right)^{2}}-\frac{3 b}{2 \omega_{1}^{5} \Omega_{0}^{4}}\right)^{2}+\left(\frac{4 \mu \omega_{1}}{3 b}\right)^{2} .
\end{aligned}
$$

$$
\begin{gathered}
\frac{F^{2} \eta^{4}}{2\left(\eta^{2} \omega_{1}^{2}-1\right)^{2}}+\frac{4 \omega_{1} \sigma}{3 b}-\frac{2 F^{2} \eta^{4}}{\left(\eta^{2} \omega_{1}^{2}-1\right)^{2}}-\frac{4}{\omega_{1}^{4} \Omega_{0}^{4}}>0 \\
\left(\frac{F^{2} \eta^{4}}{2\left(\eta^{2} \omega_{1}^{2}-1\right)^{2}}+\frac{4 \omega_{1} \sigma}{3 b}-\frac{2 F^{2} \eta^{4}}{\left(\eta^{2} \omega_{1}^{2}-1\right)^{2}}-\frac{4}{\omega_{1}^{4} \Omega_{0}^{4}}\right)^{2} \geq\left(\frac{8 \omega_{1}}{3 b}\right)^{2}\left(\frac{1}{2} \sigma-\frac{3 F^{2} \eta^{4} b}{4 \omega_{1}\left(\eta^{2} \omega_{1}^{2}-1\right)^{2}}-\frac{3 b}{2 \omega_{1}^{5} \Omega_{0}^{4}}\right)^{2}+\left(\frac{4 \mu \omega_{1}}{3 b}\right)^{2} .
\end{gathered}
$$

It shows that the subharmonic resonance of $1 / 3$ order occurs in the frequency band slightly higher than the excitation frequency. If the above inequalities are not satisfied, it means that only the steady solution, that is, the primary approximate response of the system, is the same as that of the linear system in the frequency band far away from the resonance.

\section{Numerical Verification}

In order to validate the obtained analytical results, first, the displacement responses are solved by multiple-scale method and RK method, respectively, based on the parameters of $\underline{\varepsilon}=0.01, c=0.004, \alpha_{0}=0.1, h=0.1, k_{1}=10, k_{2}=100$, and $A=1200$, and the initial conditions here are $X(0)=1$, $\dot{X}(0)=0$. It can be seen from Figure 2 that the two time-
It follows that $a^{2}=p \pm \sqrt{p^{2}-q}$, for $q>0$; thus, the necessary conditions of $1 / 3$ subharmonic resonance are $p>0, p^{2} \geq q$; that is, response curves are in good agreement, which verified the analytical solution.

The dynamic characteristics of the influence of parameters on the mechanical system are studied and the frequencyresponse curves under different heaving excitation, speed, damping are analyzed. A set of parameters of the system is selected: $\alpha_{0}=0.1, h=0.1, \mu=0.015, \omega_{1}=0.1, b=0.01, F=0.04$, $\Omega_{0}=3$, and $\eta=60$, and the frequency-response curves can be demonstrated as Figures 3-6.

By changing the frequency ratio, it can be noticed in Figure 3 that the amplitude of the frequency-response curve bends to the right and the resonance domain of the system changes as the frequency ratio $\eta$ increases. This can be potentially used for practical ship design that selecting the proper rotating speed is desired for the safety of navigation. We also notice that the resonance frequency bandwidths of 


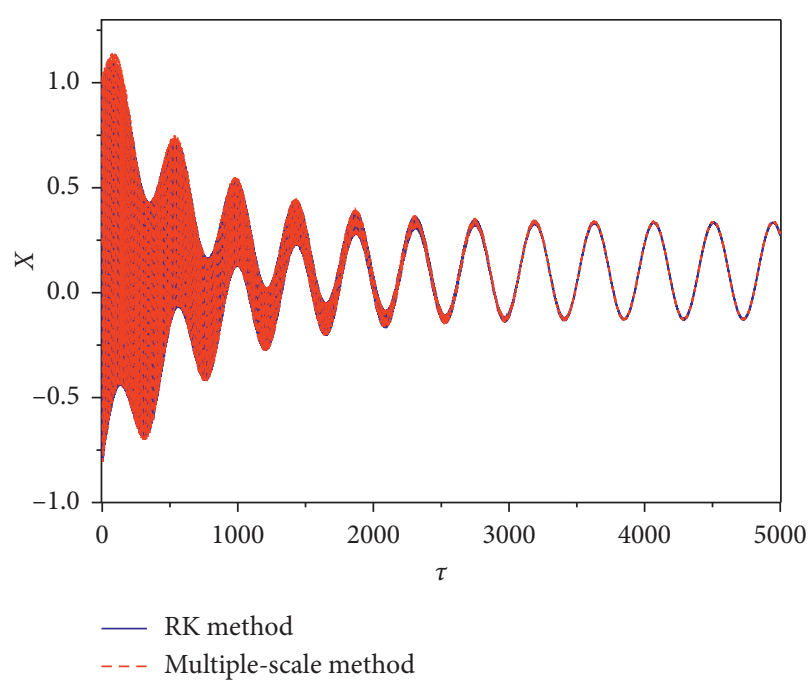

(a)

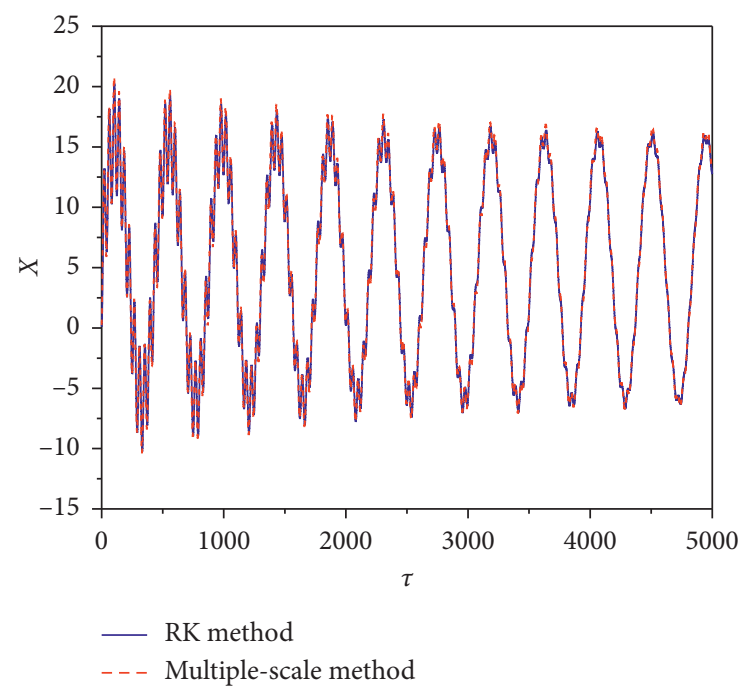

(b)

Figure 2: Time history response: the blue line for the numerical simulation; the red line for the multiple-scale method. (a) Displacement response $\left(\varepsilon=0.01, c=0.004, \alpha_{0}=0.1, h=0.1, \mu=0.3, k_{1}=10, k_{2}=100, \overline{\mathrm{A}}=1200, \Omega_{0}=3, \eta=70, X(0)=1, \dot{X}(0)=0\right)$. (b) Displacement response $\left(\varepsilon=0.01, c=0.004, \alpha_{0}=0.1, h=0.1, \mu=0.1, k_{1}=10, k_{2}=100, \overline{\mathrm{A}}=1200, \Omega_{0}=5, \eta=60, X(0)=1, \dot{X}(0)=0\right)$.

the system are affected by the excitation. And the amplitude of the frequency-response curve and the degree of bending increase with the increase of the excitation amplitude $F$. Figure 3(c) illustrates the rotor speed effects on the mechanical system. We can notice that the frequency-response curves consist of two branches: the upper branch for resonance and the lower branch for nonresonance. The increase in tuning factor does not affect the max amplitude, but the resonance frequency peaks become more and more bending, the maxima frequency shift to larger frequencies, and a jump in amplitude develops. This bending of the resonances to the right-hand side corresponds to the behavior of hardening behaviors caused by the cubic nonlinearity. Besides, the system produces three solutions in which the solid lines are the physical solution for the stable and the dashed line is the nonphysical solution for the unstable.

Additionally, $\mu$ could change the amplitude of the system as shown in Figure 3(d). When parameter $\mu$ increases, the amplitude of the curve decreases, and the interval corresponding to multivalue and jump decreases, which means the stability of the system is enhanced.

Figures 4 and 5 show the frequency-response curves of superharmonic resonance under different values of excitation, speed, and damping. It can be observed that the amplitude of the curve slightly decreases as the frequency ratio $\eta$ increases, and the bending degree and the resonance domain of the system do not change evidently, which means, in these cases, the influence of frequency ratio can be safely ignored. The results show that the amplitude of the superharmonic resonance will become larger when the frequency of the heave excitation increases, which indicates that the heave motion may cause the capsize of a ship when encountering extreme weather conditions. By closer observation, with the slight increase in the excitation amplitude $F$, the amplitude of the curve and bending degree increases sharply, but the resonance domain of the system does not change much. Figure 4(c) shows that with the increase of speed, the amplitude of the curve decreases, the frequency-response curve bends to the right, and the resonance domain of the system decreases and shifts to the left. Figure 5(c) illustrates the effects of rotor speed on frequency-response curves. The resonance can be generated when the excitation is getting closer to the natural frequency of the mechanical system. Also, we can notice that increasing the rotor speed can make the resonance frequency have a big left shift ranging from 14.99 to 31.15 , but it does not affect the max amplitude. Moreover, the damping could suppress the resonance of the system to some degree. In practical design, the vibration control of the system can be implemented by properly enlarging the damping on the airbag isolation device.

Figure 6 shows the frequency-response curve of the subharmonic resonance of $1 / 3$ order. Figure $6(a)$ indicates that the frequency curve has no obvious change with the increase of frequency ratio, and the frequency ratio $\eta$ is no longer a key influence factor of the subharmonic resonance, which is different from primary resonance. It can be observed that adding a small amount of excitation amplitude $F$ causes a shift in the frequency jump-up point. Figure 6(c) shows that, with the increase of speed $\Omega_{0}$, the overall amplitude-frequency curve shifts to the left, which means that the value of detuning factor $\sigma$ required to achieve subharmonic resonance decreases; and with the increase in damping, the peak value of resonance slightly decreases. It indicates that subharmonic resonance only occurs when the damping is small.

To further analyze the dynamic behaviors of the system, the phase diagram and Poincaré section of the equation are solved by the fourth Runge-Kutta method, and the Lyapunov exponent is solved by the method of definition [24]. Figure 7 shows that the phase plane of the system presents an 

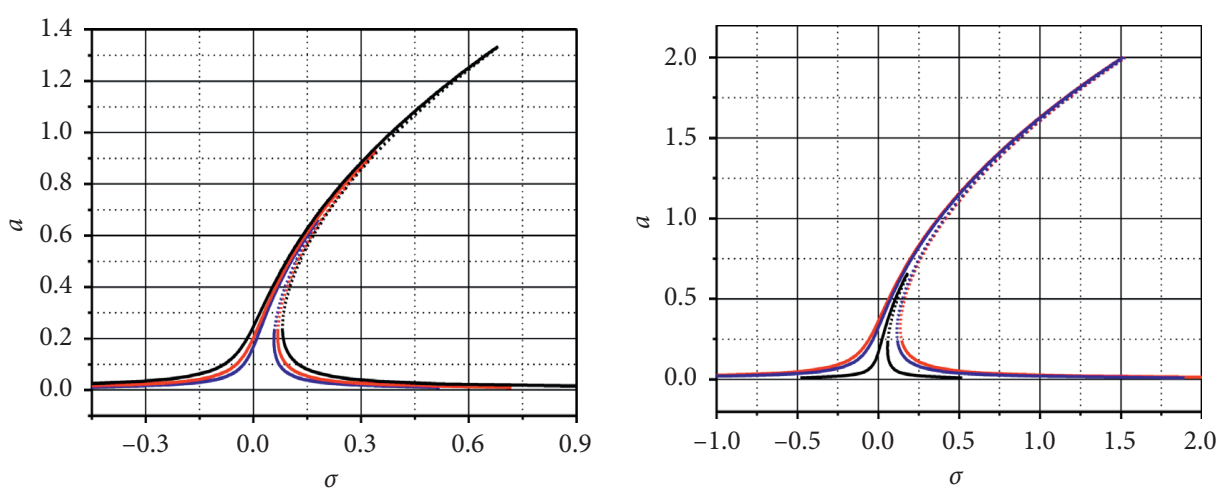

$$
\begin{aligned}
\eta & =50 \\
-\eta & =60 \\
-\eta & =70
\end{aligned}
$$

$$
\begin{aligned}
F & =0.04 \\
-F & =0.15 \\
F & =0.20
\end{aligned}
$$

(a)

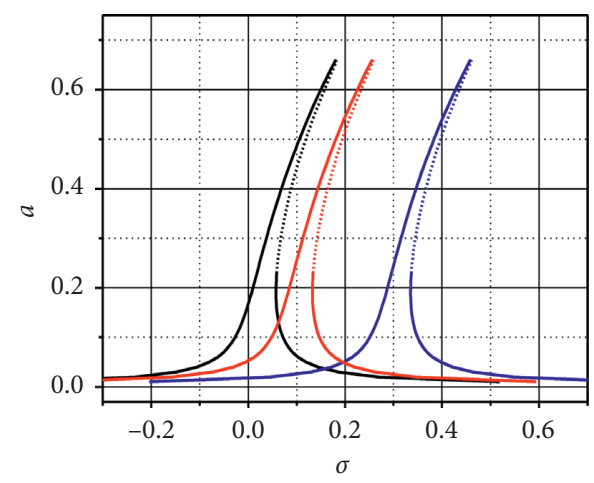

(b)

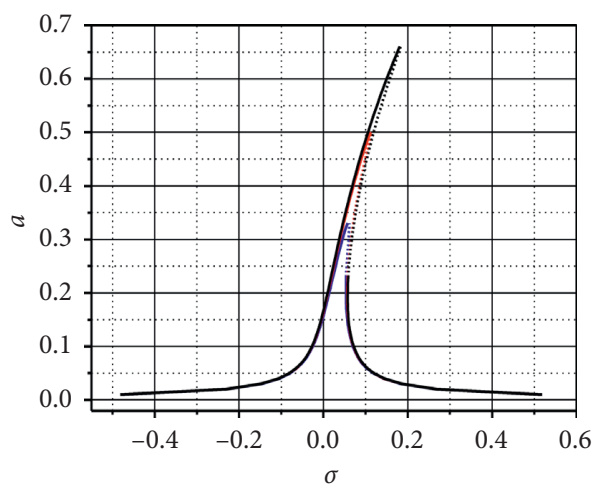

$-\Omega_{0}=1.5$

$-\Omega_{0}=2.0$

$-\Omega_{0}=3.0$

$$
\begin{aligned}
-\mu & =0.015 \\
-\mu & =0.020 \\
-\mu & =0.030
\end{aligned}
$$

(c)

(d)

FIGURE 3: Frequency-response curve of primary resonance. (a) Effects of frequency ratio. (b) Effects of excitation amplitude. (c) Effects of speed. (d) Effects of damping.

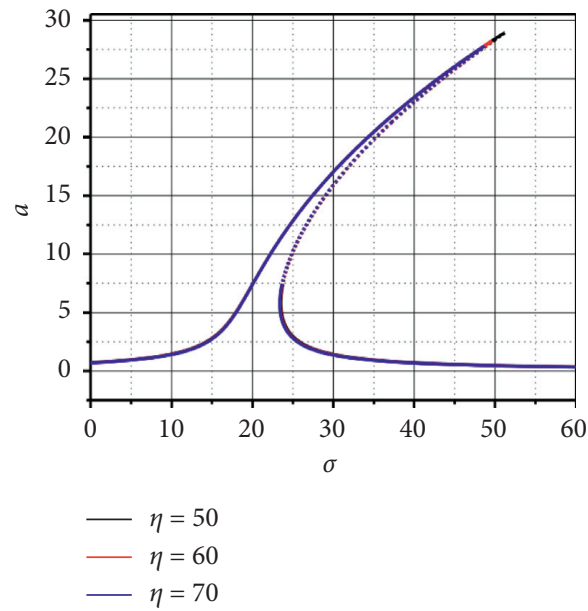

(a)

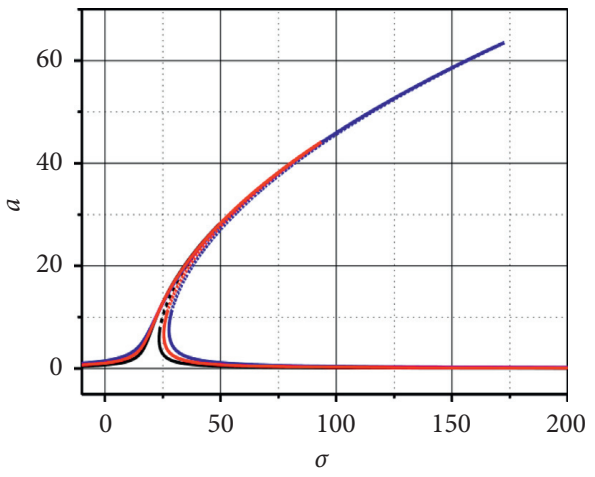

$$
\begin{aligned}
F & =0.04 \\
F & =0.05 \\
F & =0.06
\end{aligned}
$$

(b)

Figure 4: Continued. 


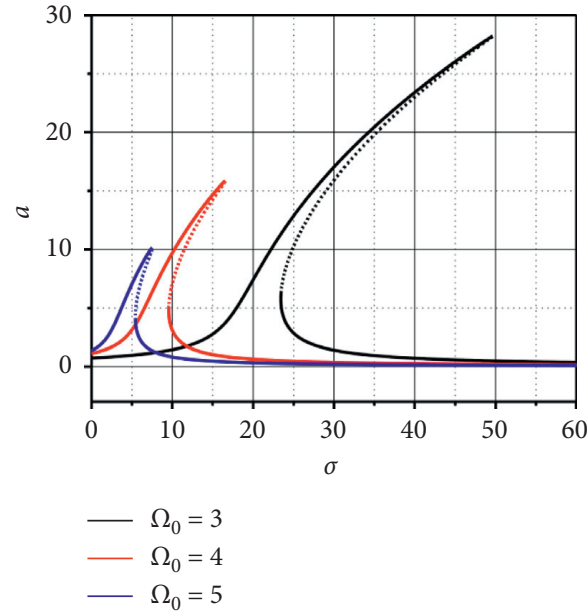

(c)

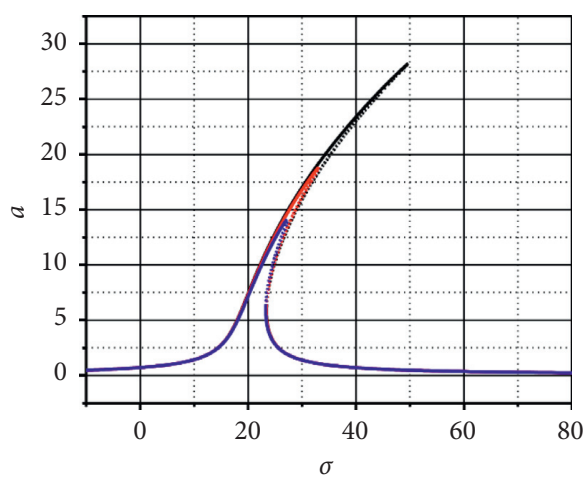

$-\mu=1.0$

$-\mu=1.5$

$-\mu=2.0$

FIGURE 4: Frequency-response curve of the superharmonic resonance of 2 orders. (a) Effects of frequency ratio. (b) Effects of excitation amplitude. (c) Effects of speed. (d) Effects of different damping.

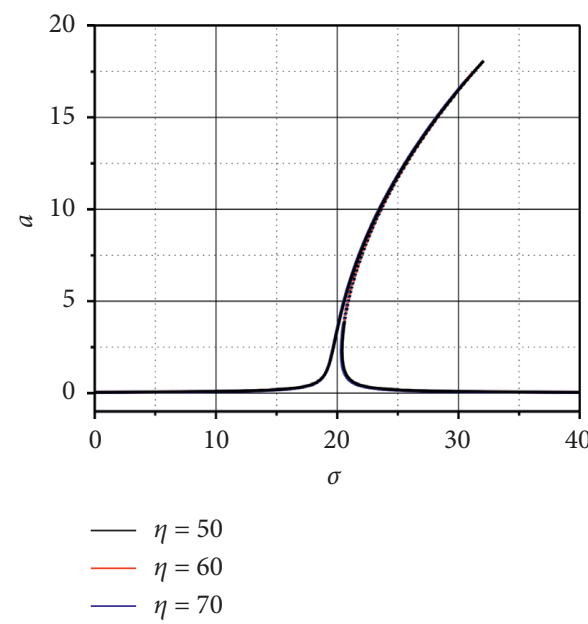

(a)

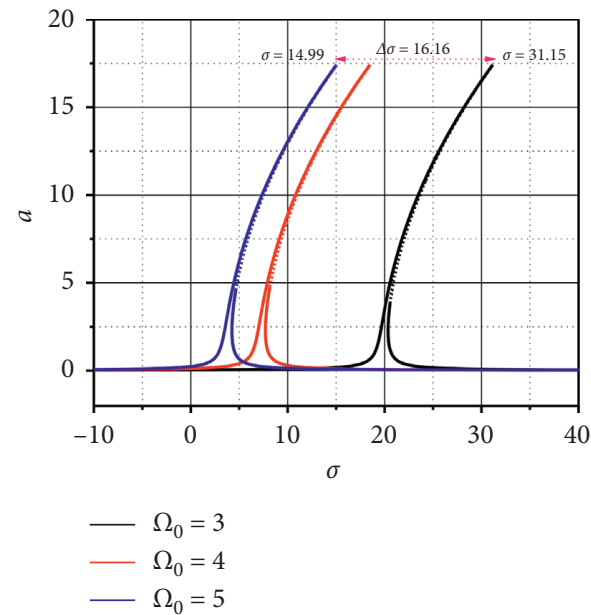

(c)

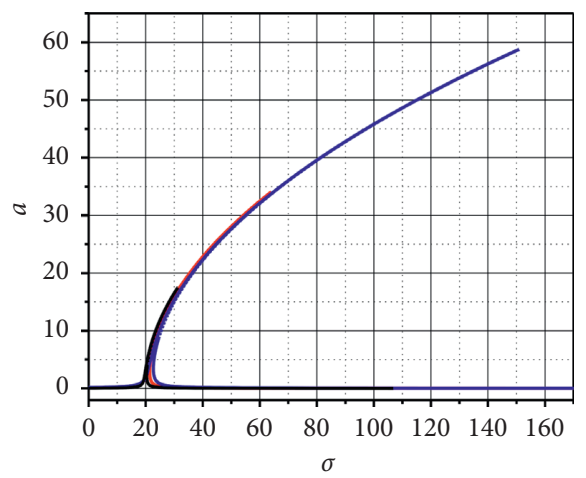

$F=0.04$

$F=0.05$

$-F=0.06$

(b)

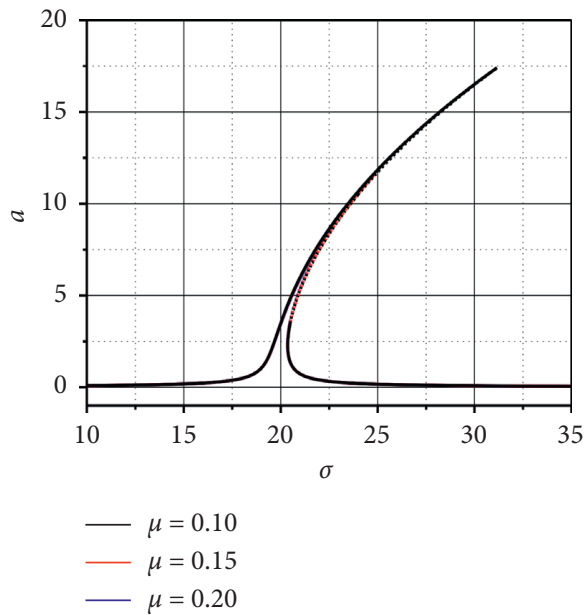

(d)

FiguRE 5: Frequency-response curve of superharmonic resonance of 3 orders. (a) Effects of frequency ratio. (b) Effects of excitation amplitude. (c) Effects of speed. (d) Effects of damping. 


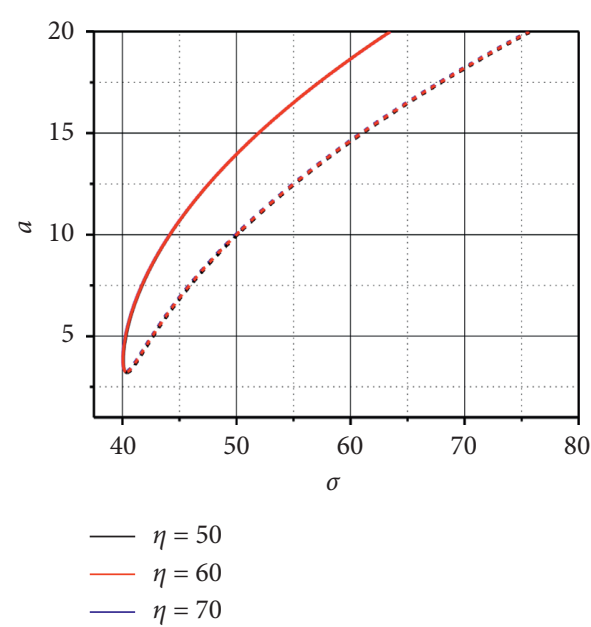

(a)

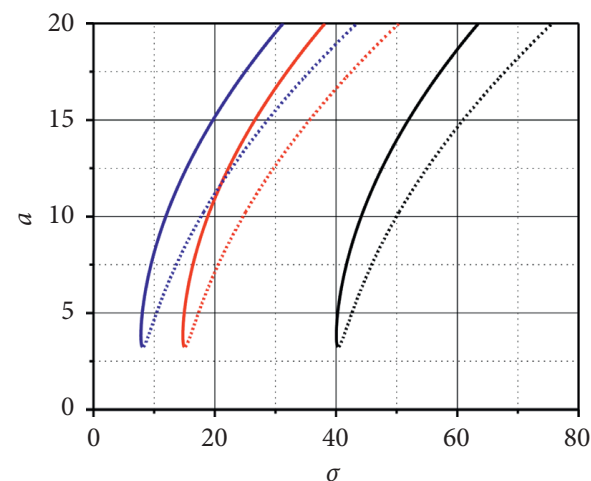

$$
\begin{array}{r}
-\Omega_{0}=3 \\
-\Omega_{0}=4 \\
-\Omega_{0}=5
\end{array}
$$

(c)

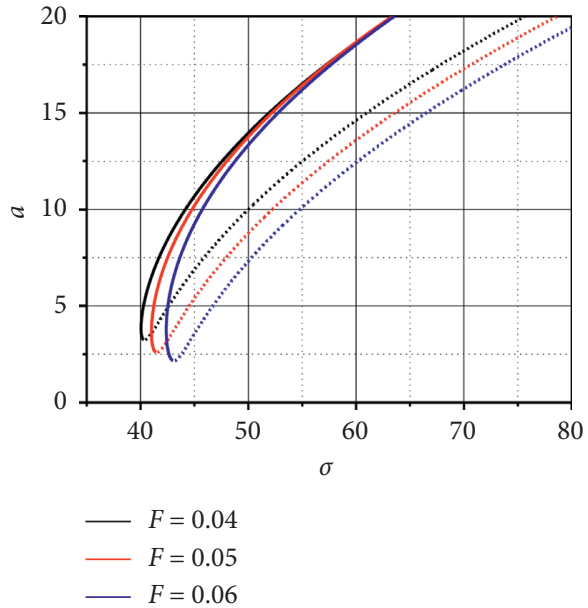

(b)

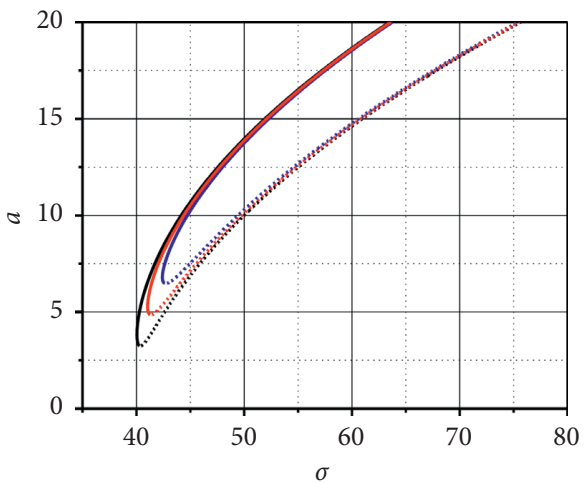

$\begin{aligned}-\mu & =1.0 \\ \mu & =1.5 \\ \mu & =2.0\end{aligned}$

(d)

FIgURE 6: Frequency-response curve of subharmonic resonance of $1 / 3$ order. (a) Effects of frequency ratio. (b) Effects of excitation amplitude. (c) Effects of speed. (d) Effects of damping.

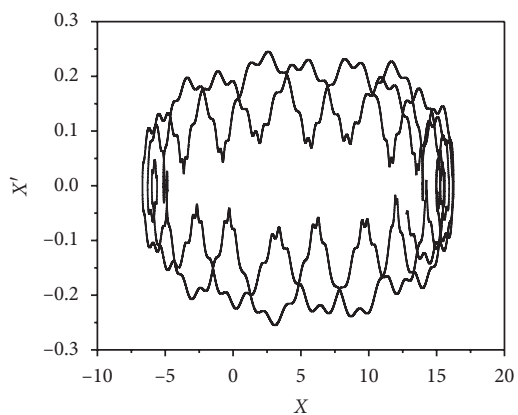

(a)

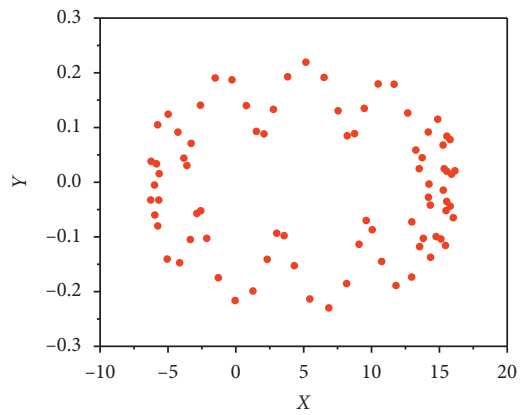

(b)

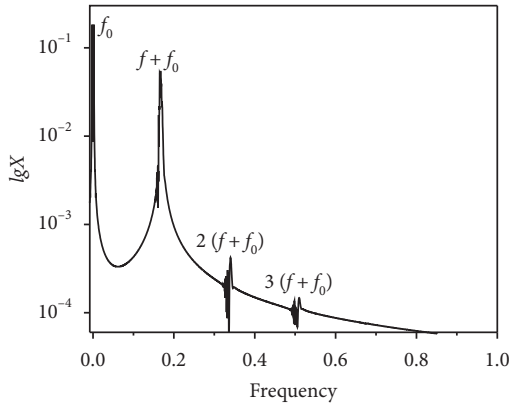

(c)

FIgURE 7: Steady-state response of the system under heaving motion. (a) Phase diagram. (b) Poincaré map. (c) Frequency spectrum.

irregular track, the Poincaré section composed of mess points is an unclosed curve, and the combined frequency $\left(f+f_{0}\right)$ and its multiple frequencies are generated in the spectrum. Figures 8 and 9 show that the time history, phase plane, and the Poincaré map of the system present a chaos state. Figure 10 shows the bifurcation diagram and the largest Lyapunov exponent diagram of the system at speed and frequency ratio. The system behaves in chaotic motion at low speed as shown in Figure 10(a) and the corresponding largest Lyapunov exponent is positive. Also, the quasiperiodic and chaos of the system appear alternately at high speed. Figure 10(b) shows that when the frequency ratio is 


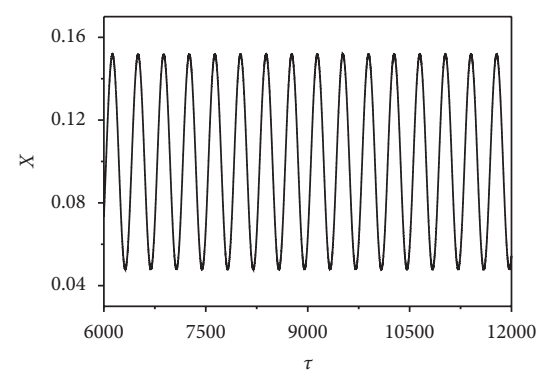

(a)

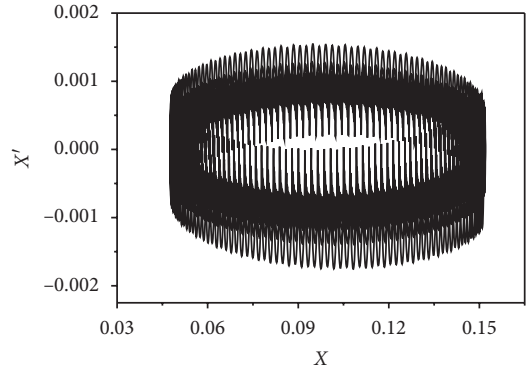

(b)

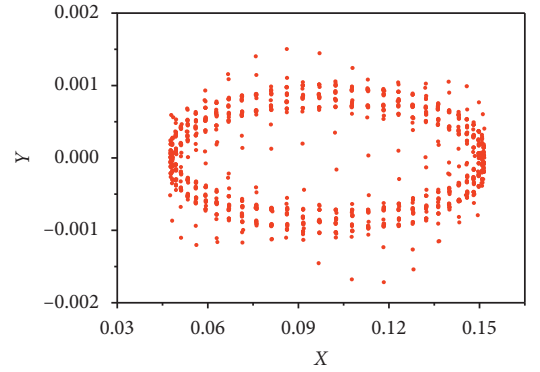

(c)

FigURE 8: Steady-state response of the system under the case of $\Omega_{0}=1.25$. (a) Displacement response. (b) Phase diagram. (c) Poincaré map.

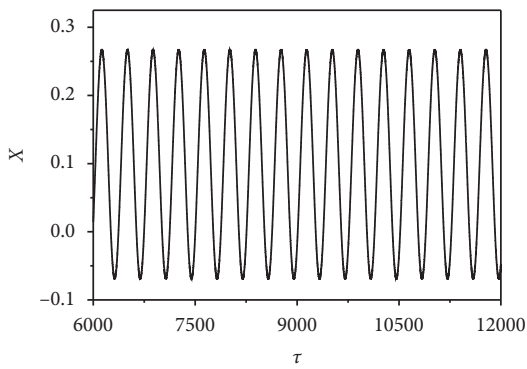

(a)

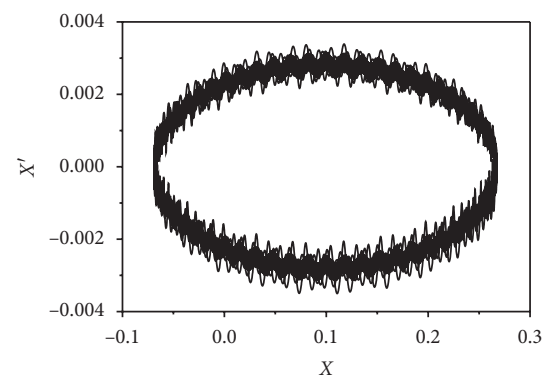

(b)

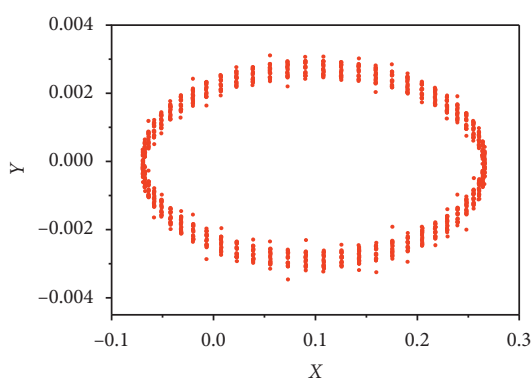

(c)

Figure 9: Steady-state response of the system under the case of $\Omega_{0}=2.25$. (a) Displacement response. (b) Phase diagram. (c) Poincaré map.

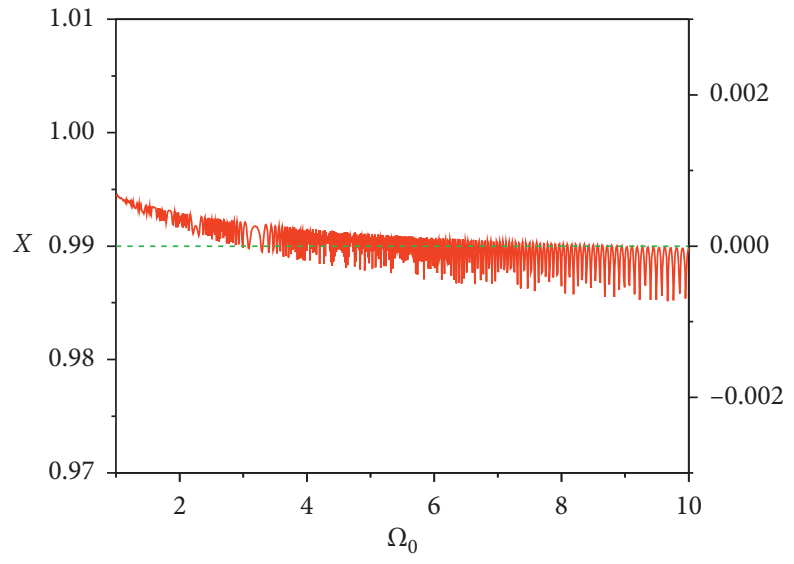

(a)

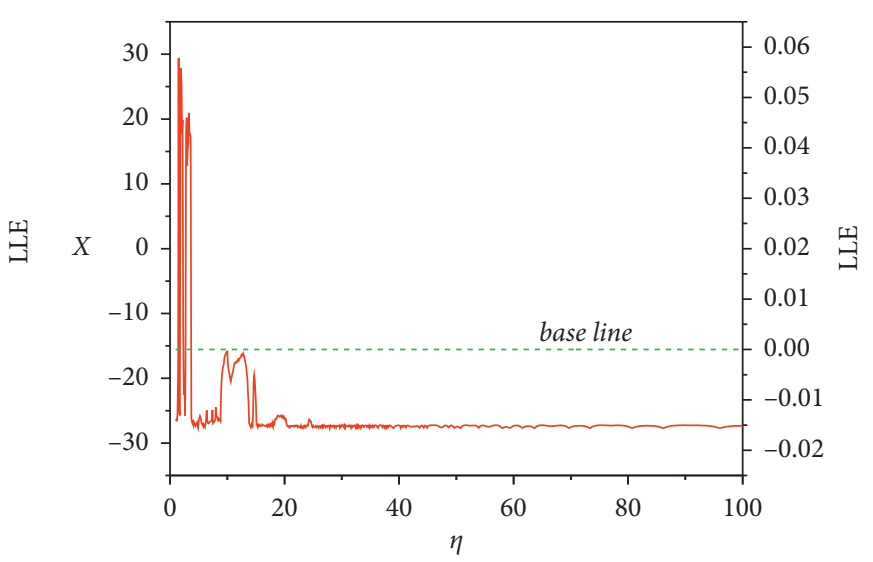

(b)

FIGURE 10: Bifurcation and LLE of the system under heaving motion. (a) Bifurcation and LLE versus speed. (b) Bifurcation and LLE versus frequency ratio. 


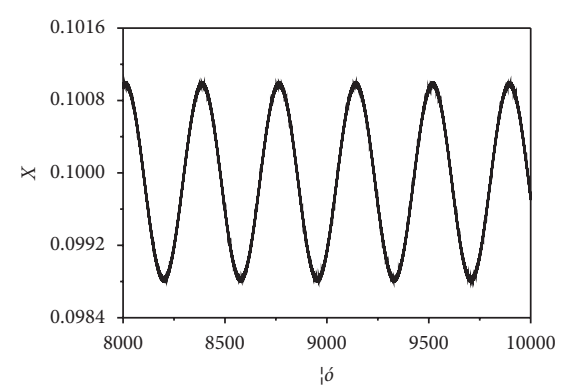

(a)

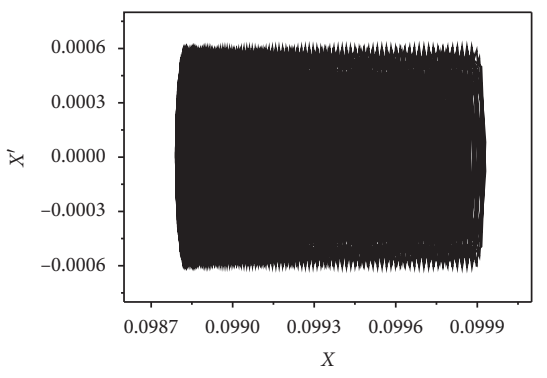

(b)

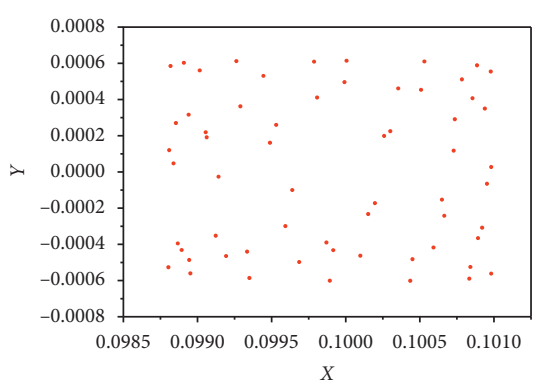

(c)

Figure 11: The response of the system under the case of $\Omega_{0}=0.18$. (a) Displacement response. (b) Phase diagram. (c) Poincaré map.

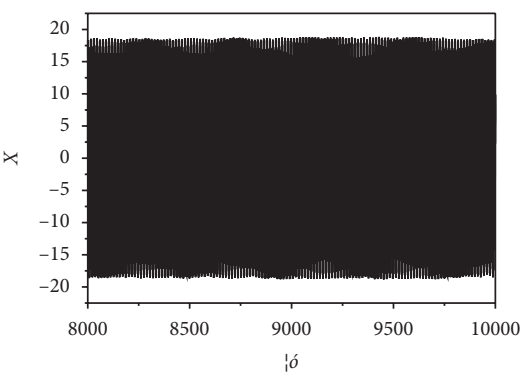

(a)

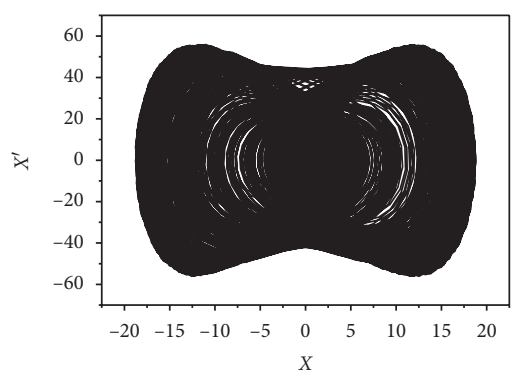

(b)

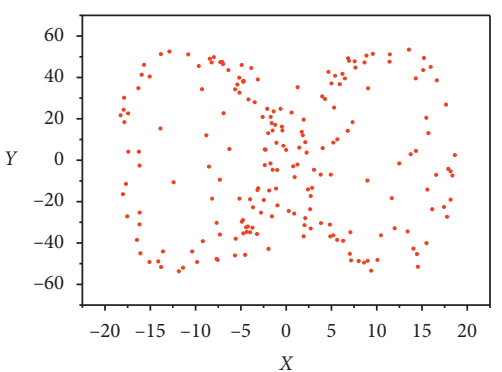

(c)

Figure 12: The response of the system under the case of $\eta=2.37$. (a) Displacement response. (b) Phase diagram. (c) Poincaré map.

less than 5 , the vibration amplitude of the system is large and the Lyapunov index is positive, which indicates the system is in a chaos state. And the system is in a quasiperiodic state when the frequency ratio is about 10 to 15 . With the increase of the frequency ratio, the amplitude of the system decreases, and the largest Lyapunov index is positive at a low-frequency ratio and becomes negative when the frequency ratio is 20 , indicating that the system tends to a stable periodic state, and the motion state can be summarized as chaos $\longrightarrow$ quasiperiodic state $\longrightarrow$ periodic state. The time history, phase diagram, and Poincaré map of the Lyapunov exponent under the greatest exponent cases of omega and eta are presented as in Figures 11 and $12(\Omega 0=0.18$ and $\eta=2.37$ ), which show the system is in chaos.

\section{Conclusions}

Based on Newton's law, the nonlinear dynamic model of the marine rotating machinery with airbag isolation device under heaving motion is established. Firstly, the approximate analytical solution of the system is studied by using the multiscale method. The stability of the system at the steady solution and the amplitude frequency-response characteristics of the system under different parameters are analyzed. Then, the nonlinear dynamic characteristics of the system are discussed by the RK method. The results can be concluded:

(1) Under the heave excitation, the steady-state motion amplitude of marine rotating machinery has the characteristics of multivalue and jump in a certain frequency range. For the primary resonance of the system, the amplitude of heave has a great influence on the stability of the system, and damping can effectively suppress the peak value of the primary resonance.

(2) For the harmonic resonance of the system, the frequency ratio is no longer a significant influence factor, which is different from the case of primary resonance. And as the excitation amplitude increases, the unstable region increases and the jump phenomenon is more apparent. And properly enlarging the damping can effectively restrain the resonance peak.

(3) The numerical results show that, with the increase in rotating speed, the system is mainly quasiperiodic and chaotic motion under the heaving motion, which has complex nonlinear characteristics. With the increase in frequency ratio, indicating that the difference between rotor frequency and heave frequency becomes larger, the system gradually tends to stable periodic motion state from unstable chaos and quasiperiodic state.

\section{Data Availability}

The raw data used to support the findings of this study are available from the corresponding author upon request.

\section{Conflicts of Interest}

The authors declare that there are no conflicts of interest regarding the publication of this paper. 


\section{Acknowledgments}

This work was supported by the National Natural Science Foundation of China (Grant no. 11972282) and the Natural Science Foundation of Shaanxi Province, China (Grant no. 2018JZ1001). This support is gratefully acknowledged.

\section{References}

[1] H. Lin and Z. Ying-Long, "Theory and design of high-pressure and heavy-duty air spring for naval vessels," Journal of Vibration Engineering, vol. 26, no. 6, pp. 886-894, 2013.

[2] A. H. Nayfeh, D. T. Mook, and L. R. Marshall, "Perturbationenergy approach for the development of the nonlinear equations of ship motion," Journal of Hydronautics, vol. 8, no. 4, pp. 130-136, 1974.

[3] B. W. Nam, Y. Kim, D. W. Kim, and Y. S. Kim, "Experimental and numerical studies on ship motion responses coupled with sloshing in waves," Journal of Ship Research, vol. 53, no. 2, pp. 68-82, 2009.

[4] S. Lee, J. M. You, and H. H. Lee, "Experimental study on the six degree-of-freedom motions of a damaged ship floating in regular waves," Journal of Oceanic Engineering, vol. 41, no. 1, pp. 40-49, 2016.

[5] L. Wang, C. Guo, Y. Su, P. Xu, and T. Wu, "Numerical analysis of a propeller during heave motion in cavitating flow," Applied Ocean Research, vol. 66, pp. 131-145, 2017.

[6] J. R. Saripilli and D. Sen, "Numerical studies on effects of slosh coupling on ship motions and derived slosh loads," Applied Ocean Research, vol. 76, pp. 71-87, 2018.

[7] J. Sanchez-Mondragon, A. O. Vázquez-Hernández, S. K. Cho, and H. G. Sung, "Yaw motion analysis of a FPSO turret mooring system under wave drift forces," Applied Ocean Research, vol. 74, pp. 170-187, 2018.

[8] L. Xiu-He, Z. Cai-Chao, F. Zhi-Xin, C. Xu, and T. Jian-Jun, "Effects of the yaw error and the wind-wave misalignment on the dynamic characteristics of the floating offshore wind turbine," Ocean Engineering, vol. 199, Article ID 106960, 2020.

[9] Z. Chang-Sheng and C. Yong-Jun, "General dynamic model of aeroengine's rotor system during maneuvering flight," Aerospace Power, vol. 24, pp. 371-377, 2009.

[10] H. Lei and C. Yushu, "Dynamical simulation and load control of a Jeffcott rotor system in Herbst maneuvering flight," Journal of Vibration and Control, vol. 22, no. 2, pp. 412-425, 2016.

[11] H. Lei, C. Yu-Shu, C. Qing-Jie, and L. Zheng-Yong, "Nonlinear vibration analysis of a cracked rotorball bearing system during flight maneuvers," Mechanism and Machine Theory, vol. 105, pp. 515-528, 2016.

[12] W. Lin-Peng, Y. Dai, and C. Yang, "Gust response analysis for helicopter rotors in the hover and forward flights," Shock and Vibration, vol. 2017, no. 4, pp. 1-20, Article ID 8986217, 2017.

[13] W. Zheng-Hao, Y. Hui-Qun, F. Gai-Yan, M. Qing-Xin, and S. Cheng-Yan, "Influence of bearing random excitation on random response of rotor system," Journal of Vibration and Shock, vol. 32, no. 17, pp. 113-117, 2013.

[14] M. Dakel, S. Baguet, and R. Dufour, "Nonlinear dynamics of a support-excited flexible rotor with hydrodynamic journal bearings," Journal of Sound and Vibration, vol. 333, no. 10, pp. 2774-2799, 2014.

[15] N. A. Saeed and H. A. El-Gohary, "On the nonlinear oscillations of a horizontally supported Jeffcott rotor with a nonlinear restoring force," Nonlinear Dynamics, vol. 88, no. 1, pp. 293-314, 2017.
[16] Z. Guanghui, L. Shupeng, M. Ruixian, and L. Zhansheng, "Nonlinear dynamic characteristics of journal bearing-rotor system considering the pitching and rolling motion for marine turbo machinery," Proceedings of the Institution of Mechanical Engineers, Part M: Journal of Engineering for the Maritime Environment, vol. 229, no. 1, pp. 95-107, 2015.

[17] L. Zhen-Xing, L. Zhan-Sheng, Y. Xiang-Yu, and P. Xue-Jia, "Dynamic analysis of journal bearing-gear system under swing movement of the ship," Journal of Mechanical Engineering, vol. 54, no. 17, p. 226, 2018.

[18] H. Yong-Chao and L. Ming, "Nonlinear dynamic characteristics of marine rotor-bearing system under heaving motion," Shock and Vibration, vol. 2019, no. 1, Article ID 7683952, 2019.

[19] Z. Wen, L. Ming, and L. Yuanbo, "Nonlinear dynamics of marine rotor system coupled with air bag-floating raft subjected to the basement excitations in lateral directions," Shock and Vibration, vol. 43, no. 1, pp. 1-20, 2020.

[20] Z. Wen, L. Ming, and X. Ling, "Nonlinear dynamic behaviors of a marine rotor-bearing system coupled with air bag and floating-raft," Shock and Vibration, vol. 18, no. 5, pp. 620968.1-620968.18, 2015.

[21] T. Soni, J. K. Dutt, and A. S. Das, "Magnetic bearings for marine rotor systems-effect of standard ship maneuver," IEEE Transactions on Industrial Electronics, vol. 99, no. 1, 2020.

[22] Y. U. Yin, H. U. Yu-Ren, and J. Xian-Ding, "Current situation and trends in some aspects of research on nonlinear rolling of ships in waves," Journal of Ship Mechanics, vol. 34, pp. 73-77, 2000.

[23] C. Liao, Z. Kongkang, and L. Zhongxing, "Dynamic characteristics fitting of air springs and numerical analysis of air suspensions with variant stiffness," Journal of Mechanical Engineering, vol. 46, no. 4, pp. 93-98, 2010.

[24] A. Wolf, H. L. Swift, and J. A. Vastano, "Determining Lyapunov exponents from a time series," Physica D: Nonlinear Phenomena, vol. 16, no. 3, pp. 285-317, 1985. 